\title{
From stable to unstable anomaly-induced inflation
}

\author{
Tibério de Paula Netto ${ }^{1, a}$, Ana M. Pelinson ${ }^{2, b}$, Ilya L. Shapiro ${ }^{1,3, c}$, Alexei A. Starobinsky , $^{4,5}$ \\ ${ }^{1}$ Departamento de Física, ICE, Universidade Federal de Juiz de Fora, Campus Universitário, Juiz de Fora, MG 36036-330, Brazil \\ ${ }^{2}$ Departamento de Física, CFM, Universidade Federal de Santa Catarina, Bairro da Trindade, Caixa Postal 476, Florianópolis, SC 88040-970, \\ Brazil \\ 3 Tomsk State Pedagogical University and Tomsk State University, Tomsk, Russia \\ ${ }^{4}$ L. D. Landau Institute for Theoretical Physics RAS, Moscow 119334, Russia \\ ${ }^{5}$ Department of Physics and Astronomy, Institute for Theoretical Physics, Utrecht University, 3508 TD Utrecht, The Netherlands
}

Received: 5 October 2015 / Accepted: 20 September 2016 / Published online: 6 October 2016

(C) The Author(s) 2016. This article is published with open access at Springerlink.com

\begin{abstract}
Quantum effects derived through conformal anomaly lead to an inflationary model that can be either stable or unstable. The unstable version requires a large dimensionless coefficient of about $5 \times 10^{8}$ in front of the $R^{2}$ term that results in the inflationary regime in the $R+R^{2}$ ("Starobinsky") model being a generic intermediate attractor. In this case the non-local terms in the effective action are practically irrelevant, and there is a 'graceful exit' to a low curvature matter-like dominated stage driven by high-frequency oscillations of $R$ - scalarons, which later decay to pairs of all particles and antiparticles, with the amount of primordial scalar (density) perturbations required by observations. The stable version is a genuine generic attractor, so there is no exit from it. We discuss a possible transition from stable to unstable phases of inflation. It is shown that this transition is automatic if the sharp cut-off approximation is assumed for quantum corrections in the period of transition. Furthermore, we describe two different quantum mechanisms that may provide a required large $R^{2}$-term in the transition period.
\end{abstract}

\section{Introduction}

There are many solid results in quantum field theory (QFT) in curved space-time, concerning divergences and renormalization and to the evaluation of finite quantum corrections (see, for example, [1-5] for introduction and further references, and [6,7] for a recent review). The most interesting applications concern vacuum sector of the theory and the one-loop approximation is usually considered reliable.

\footnotetext{
a e-mail: tiberiop@fisica.ufjf.br

b e-mail: ana.pelinson@gmail.com

c e-mail: shapiro@fisica.ufjf.br

de-mail: alstar@landau.ac.ru
}

Hence the main interest is usually paid to the quantum effects of free matter fields on an arbitrary classical gravitational background. In particular, for the case of free massless conformal fields in a Friedmann-Lemaitre-Robertson-Walker (FLRW) isotropic cosmological model, an explicit calculation of the finite average value of the energy-momentum tensor (EMT) of these fields is possible by using the conformal anomaly [8,9] (see also the earlier pioneer references $[10,11]$ on the EMT regularization and calculation in a more general anisotropic cosmology, and [12] for a general and historical review). The early work concerning cosmological applications of the conformal anomaly $[13,14]$ led to the first inflationary model $[15,16]$, which was extensively studied (see, e.g., [17-23]), including inhomogeneous perturbations of this modified gravity model in the scalar [24-26] and tensor $[25,26]$ sectors (following the pioneer calculation of the generation of tensor perturbations during inflation in the case of Einstein gravity in [27]).

The anomaly-induced effective action in $d=4$ was first calculated in [28,29] (see also [14] for the earlier non-covariant version and [30-32] for a more complete local covariant presentation), similar to the famous Polyakov action [33] in $d=2$. The application of this effective action to cosmology was first considered in [34], where the possible torsion terms were also taken into account. Later on, the effective action approach was systematically pursued in $[35,36]$. The main advantage of using the anomaly-induced effective action is a better control of the approximations and also a better starting point for possible generalizations.

Anomaly-driven inflation can be stable or unstable, depending on the sign of the local $R^{2}$-term [15,16,37]. If such a term is not introduced at the classical level, the stability depends on the number of particles of different spin $(0$, $1 / 2$ and 1) in the underlying QFT on curved space-time background. In particular, for the supersymmetric particle con- 
tent inflation is stable and, for the Minimal Standard Model (MSM) of particle physics, it is unstable [38]. It is possible to have inflation which starts as stable due to supersymmetry. After some time supersymmetry breaks down and inflation becomes unstable. The reason why supersymmetry can disappear is related to the greater masses of the $s$-particles that decouple according to the Appelquist and Carazzone decoupling theorem [39]. Let us note that the gravitational version of the decoupling theorem has been derived in $[40,41]$, hence the described scheme looks consistent with the well-known QFT results.

A relevant question is why the energy scale of stable inflation is decreasing, such that the gravitational decoupling could take place. The solution to this problem has been suggested in [42-44]. The stable anomaly-induced inflation is due to the quantum effects of massless conformal fields, and is strictly exponential, such that the Hubble parameter is constant. However, taking the weak effects of the masses of the quantum fields into account, one can observe a tempered form of inflation, with decreasing magnitude of the Hubble parameter.

The second interesting question is what happens with the universe after it leaves the stable inflationary stage. For the choice of parameters which corresponds to the unstable inflation, there are different types of solutions $[15,16]$. The desirable one is when the universe is asymptotically approaching the FRW-behavior. Then the non-local part of anomalyinduced action rapidly becomes irrelevant and the evolution is essentially driven by the local $R^{2}$ term. Moreover, in order to control cosmic perturbations after inflation, the coefficient of this term must be very large, of the order of $5 \times 10^{8}$ [26]. This type of inflationary model is supported by all known observations, including Planck data [45].

At the same time, there are other solutions in the theory with anomaly-induced corrections, which can be called hyperinflation [35]. In this case the expansion of the universe is even more violent than in the exponential phase, and there is no chance for a sound physical interpretation of such a solution. The first purpose of the present work is to see which of the two possible scenarios of the post-stable evolution takes place. The simplest possible approach in used. Namely, we assume that the unstable phase starts exactly at the point where the stable phase ends. Another important issue discussed in this paper concerns quantum mechanisms to generate a large coefficient of the $R^{2}$-term in the transition period from stable to unstable inflation. We demonstrate that this effect may take place because of a possible strong coupling between fields which may result in a large value of the parameters $\xi$ of the non-minimal interaction of scalar fields with scalar curvature.

The paper is organized as follows. In order to have a selfconsistent presentation, Sect. 2 includes a brief review of the effective action induced by anomaly, and also the inflationary solutions, both stable and unstable, including tempered stable inflation due to the effects of massive fields. The difficulties of the QFT-based systematic study of the transition period are also briefly explained. Section 3 describes the numerical results concerning the transition between stable and unstable epochs in the sharp cut-off approximation. In Sect. 4 these results are supported by analysis of the phase diagrams in both cases. Section 5 describes two alternative (but related) quantum mechanisms to generate a large coefficient of the $R^{2}$-term in the transition epoch. Finally, in the last section we draw our conclusions and discuss further perspectives of the QFT-based approach to inflation.

\section{Brief review of anomaly-driven inflation}

The effective action of vacuum is defined through the path integral over the set of all matter fields $\Phi$, including gauge fields and ghosts (e.g., in Standard Model or GUTs), ${ }^{1}$

$e^{i \Gamma\left(g_{\mu v}\right)}=\int \mathrm{d} \Phi e^{i S\left(\Phi, g_{\mu v}\right)}$.

The consistency of the theory requires that the classical action includes vacuum part, $S\left(\Phi, g_{\mu \nu}\right)=S_{\text {vacuum }}\left(g_{\mu \nu}\right)+$ $S_{\text {matter }}\left(\Phi, g_{\mu \nu}\right)$, where the first term does not depend on the matter fields, but still has to be renormalized. The vacuum action of renormalizable theory should include EinsteinHilbert term with a cosmological constant,

$S_{\mathrm{EH}}=-\frac{1}{16 \pi G} \int \mathrm{d}^{4} x \sqrt{-g}(R+2 \Lambda)$

and four covariant four-derivative terms,

$S_{\mathrm{HD}}=\int \mathrm{d}^{4} x \sqrt{-g}\left\{a_{1} C^{2}+a_{2} E+a_{3} \square R+a_{4} R^{2}\right\}$.

Here $a_{1}, \ldots, a_{4}$ are parameters of the vacuum action. In the conformal case one can set $a_{4}=0$, but it is also possible to include this term. The full action of the external metric is

$S_{\text {vacuum }}=S_{\mathrm{HD}}+S_{\mathrm{EH}}$.

Since gravity is not quantized, there is no problem with unitarity of gravitational $S$-matrix. Instead, one should worry about the stability of the approximate low-energy classical solutions below Planck energy scale, as discussed recently in $[46,47]$.

\subsection{Anomaly and induced effective action}

In the very early universe the masses of quantum fields and their interactions are assumed to be irrelevant. Consider conformal theory with $N_{s}$ real scalars, $N_{f}$ Dirac fermions and

\footnotetext{
${ }^{1}$ Our notations are $\eta_{\mu \nu}=\operatorname{diag}(+---)$ and $R_{\mu \nu}=\partial_{\lambda} \Gamma_{\mu \nu}^{\lambda}-\cdots$.
} 
$N_{v}$ massless vectors. For the scalar massless fields $\varphi$ conformal invariance requires that the non-minimal parameters of the $\xi R \varphi^{2}$-interaction are $\xi=1 / 6$. Taking $a_{4}=0$, the action $S_{H D}$ satisfies the conformal Noether identity

$-\frac{2}{\sqrt{-g}} g_{\mu \nu} \frac{\delta S_{H D}}{\delta g_{\mu \nu}}=0$,

which means zero trace for the stress tensor of vacuum $T_{\mu}^{\mu}=0$. At the quantum level, this condition is violated by an anomaly,

$$
\begin{aligned}
T & =\left\langle T_{\mu}^{\mu}\right\rangle=-\frac{2}{\sqrt{-g}} g_{\mu \nu} \frac{\delta \bar{\Gamma}^{(1)}}{\delta g_{\mu \nu}} \\
& =-\left(w C^{2}+b E+c \square R\right),
\end{aligned}
$$

where $w, b$, and $c$ are $\beta$-functions for the parameters $a_{1}, a_{2}, a_{3}$, which depend on the number of (real) scalar, (Dirac) spinor, and gauge vector fields, $N_{s}, N_{f}, N_{v}$,

$w=\beta_{1}=\frac{1}{(4 \pi)^{2}}\left(\frac{N_{s}}{120}+\frac{N_{f}}{20}+\frac{N_{v}}{10}\right)$,

$b=\beta_{2}=-\frac{1}{(4 \pi)^{2}}\left(\frac{N_{s}}{360}+\frac{11 N_{f}}{360}+\frac{31 N_{v}}{180}\right)$,

$c=\beta_{3}=\frac{1}{(4 \pi)^{2}}\left(\frac{N_{s}}{180}+\frac{N_{f}}{30}-\frac{N_{v}}{10}\right)$.

One has to note that the coefficient $c$ has the well-known regularization-dependent ambiguity, which is equivalent to the possibility to add the $a_{4} R^{2}$-term at the classical level (see, e.g., [12]). This issue was discussed in full details in [48], using both dimensional and covariant Pauli-Villars regularization. It was shown that the ambiguity concerns the starting point of the renormalization group trajectory and not the flow itself. In particular, this means one can fix it by imposing a renormalization condition on the classical coefficient $a_{4}$. There is nothing wrong in defining the $R^{2}$ term by hand, but it is more natural to assume that this term comes from vacuum quantum effects according to (6), which corresponds to the point-splitting [49] and $\zeta$ regularizations [50]. ${ }^{2}$ In Sect. 5 we shall discuss the possibility of significant change of the overall coefficient $a_{4}$ of the $R^{2}$-term in the epoch of transition from stable to unstable inflation.

Natural question concerns possible effect of higher loops. Let us remember that the non-perturbative structure of conformal anomaly is basically the same as at one loop. This statement is known as $a$ - and $c$-theorems and gained significant attention in the recent years [51-53]. Since only the trace anomaly is relevant for the dynamics of conformal factor, one

\footnotetext{
2 The equivalent n-wave and adiabatic EMT regularizations proposed earlier in $[10,11]$ respectively lead to the same result for conformal anomaly if applied to the case of a non-zero rest mass $m$ of a quantum field with $m$ set to zero in the final result.
}

can safely assume that at higher loops nothing changes dramatically and conclude that the one-loop approximation is sufficiently reliable in this case.

The one-loop part $\bar{\Gamma}^{(1)}$ of the vacuum effective action satisfies the equation

$$
-\frac{2}{\sqrt{-g}} g_{\mu \nu} \frac{\delta \bar{\Gamma}^{(1)}}{\delta g_{\mu \nu}}=\left\langle T_{\mu}^{\mu}\right\rangle,
$$

which can be solved in the form $[28,29]$

$$
\begin{aligned}
\Gamma_{\text {ind }}= & S_{c}\left[\bar{g}_{\mu \nu}\right] \\
& +\int d^{4} x \sqrt{-\bar{g}}\left\{w \sigma \bar{C}^{2}+b \sigma\left(\bar{E}-\frac{2}{3} \bar{\square} \bar{R}\right)+2 b \sigma \bar{\Delta}_{4} \sigma\right. \\
& \left.-\frac{1}{12}\left(c+\frac{2}{3} b\right)\left[\bar{R}-6(\bar{\nabla} \sigma)^{2}-6 \bar{\square} \sigma\right]^{2}\right\}
\end{aligned}
$$

where we separated the conformal degree of freedom $\sigma$ according to

$g_{\mu \nu}=\bar{g}_{\mu \nu} \cdot e^{2 \sigma(x)}=\bar{g}_{\mu \nu} \cdot a^{2}(x)$

and used notation

$\Delta_{4}=\square^{2}+2 R^{\mu \nu} \nabla_{\mu} \nabla_{\nu}-\frac{2}{3} R \square+\frac{1}{3}\left(\nabla^{\mu} R\right) \nabla_{\mu}$

for the fourth derivative, conformal operator acting on the conformal-invariant scalars. The term $S_{c}$ in (11) is a conformal-invariant functional, $S_{c}\left[\bar{g}_{\mu \nu}\right]=S_{c}\left[g_{\mu \nu}\right]$, which is an "integration constant" for Eq. (10). In cosmology, this term is irrelevant for defining the dynamics of the conformal factor of the background metric, $a(\eta)$, and therefore (11) is the exact form of quantum correction in this case. The general fiducial metric is

$\mathrm{d} \bar{s}^{2}=\bar{g}_{\mu \nu} \mathrm{d} x^{\mu} \mathrm{d} x^{\nu}=\mathrm{d} \eta^{2}-\frac{\mathrm{d} r^{2}}{1-k r^{2}}-r^{2} \mathrm{~d} \Omega$,

where $\eta$ is the conformal time. In the following we consider a spatially flat metric, $k=0$.

\subsection{Stable and unstable solutions}

The dynamics of conformal factor is defined from the variational principle of the total action, including quantum corrections,

$S_{t}=S_{\text {vacuum }}+\Gamma_{\text {ind }}$.

Then we arrive at the following equation:

$$
\begin{gathered}
\frac{\dddot{a}}{a}+\frac{3 \ddot{a} \ddot{a}}{a^{2}}+\frac{\ddot{a}^{2}}{a^{2}}-\left(5+\frac{4 b}{c}\right) \frac{\ddot{a} \dot{a}^{2}}{a^{3}} \frac{\ddot{a}}{a^{3}} \\
-\frac{M_{\mathrm{P}}^{2}}{8 \pi c}\left(\frac{\ddot{a}}{a}+\frac{\dot{a}^{2}}{a^{2}}-\frac{2 \Lambda}{3}\right)=0,
\end{gathered}
$$

where $M_{\mathrm{P}}^{2}=1 / G$ is the square of the Planck mass. We assume that the cosmological constant $\Lambda$ always satisfies the 
condition $0<\Lambda \ll M_{P}^{2}$. Equation (16) is written in terms of the physical time $t$, where $\mathrm{d} t=a(\eta) \mathrm{d} \eta$. An equivalent thirdorder equation (34) can be obtained as the 00-component of the Einstein equation with quantum corrections [14]. Moreover, this equation can be reduced to a first-order one, as shown in Sect. 4. Let us note that a more detailed discussion of deriving 00- and ij-components of generalized Friedmann equations was given in [23] and generalizations in the presence of radiation were given in [54].

Equation (16) has important particular solutions with constant Hubble parameter $[15,16]$ (for $\Lambda \neq 0$ the solution was obtained in $[43,44])$,

$a(t)=a_{0} \cdot e^{H_{0} t}$,

where

$H_{0}=H_{ \pm}=\frac{M_{\mathrm{P}}}{\sqrt{-32 \pi b}} \cdot\left(1 \pm \sqrt{1+\frac{64 \pi b}{3} \frac{\Lambda}{M_{\mathrm{P}}^{2}}}\right)^{1 / 2}$

The $H_{+}$solution is close to the original one of $[15,55]$, $H=M_{P} / \sqrt{-16 \pi b}$, which is an exponential inflationary solution. The second value is close to the classical $\mathrm{dS}$ solution $H_{-} \approx \sqrt{\Lambda / 3}$ without quantum corrections. In the following we will mainly be concerned with the inflationary phase and, therefore, assume $H_{0}=H_{+}$.

The solution (18) is real since $b<0$, according to (8). At the same time, the coefficient $c$ in Eq. (9) may have different signs, depending on the particle content of the theory. For small perturbations $\sigma(t) \rightarrow \sigma(t)+\delta \sigma(t)$ around inflationary exponential solution, it is easy to check that it is stable for $c>0$ and unstable for $c<0[15,43,44]$. Assuming (9), the stability condition $c>0$ boils down to the relation

$\frac{1}{3} N_{f}+\frac{1}{18} N_{s}>N_{v}$

satisfied for any realistic supersymmetric model. Then $H=$ $H_{+}$is the unique stable attractor and hence inflation starts for any choice of initial conditions with homogeneous and isotropic metric.

On the other hand, (19) is not satisfied for the Minimal Standard Model of Particle Physics [42]. Another case when the condition (19) is not satisfied is the present-days universe. Since the decoupling of the lightest massive particles (presumably neutrino), photon is the unique "active" quantum particle, such that $N_{v}=1$ and $N_{f}=N_{s}=0$.

Let us note that in this case the classical $\mathrm{dS}$ solution with $H=\sqrt{\Lambda / 3}$ is stable under small perturbations of Hubble parameter $[43,44]$, which is a relevant consistency test for the whole approach. The same is true for the tensor perturbations, which do not grow up for dS [23, 27,36] and for other classical solutions, even in the presence of matter or radiation $[46,47]$.

\subsection{Effect of masses and tempered stable inflation}

Consider some realistic supersymmetric model, where $s$ particles have relatively large masses. Other particles can be approximately regarded as massless.

At the beginning of the stable inflation Hubble parameter $H$ is even greater that all masses and the last can be seen as small perturbations violating conformal invariance. In this case one can apply a conformal description of the massive theory [42-44] (similar approach can be found in [56,57], see also [58]). The masses of matter fields, Newton constant and cosmological constant are replaced by powers of a new auxiliary scalar $\chi$,

$m_{s}^{2} \rightarrow \frac{m_{s}^{2}}{M^{2}} \chi^{2}$

$m_{f} \rightarrow \frac{m_{f}}{M} \chi$

$\frac{1}{16 \pi G} R \rightarrow \frac{M_{P}^{2}}{16 \pi M^{2}}\left[R \chi^{2}+6(\partial \chi)^{2}\right]$,

$\Lambda \rightarrow \frac{\Lambda}{M^{2}} \chi^{2}$

where $M$ is a new dimensional parameter. For the EinsteinHilbert term the kinetic term for $\chi$ provides conformal invariance of the action. In order to have local conformal invariance one can define the field $\chi$ to transform as

$\chi \rightarrow \chi e^{-\sigma(x)}$,

while other fields transform according to

$$
\begin{aligned}
g_{\mu \nu} & \rightarrow g_{\mu \nu} \cdot e^{2 \sigma(x)}, \\
\varphi & \rightarrow \varphi \cdot e^{-\sigma(x)}, \\
\psi & \rightarrow \psi \cdot e^{-3 \sigma(x) / 2} .
\end{aligned}
$$

Now we can calculate anomaly and anomaly-induced effective action. Finally, we fix the conformal gauge according to $\chi=\bar{\chi} e^{-\sigma}=M$. The result has the form

$$
\begin{aligned}
\Gamma^{(1)}= & S_{\mathrm{HD}}+\Gamma_{\text {ind }} \\
& -\int \mathrm{d}^{4} x \sqrt{-\bar{g}} e^{2 \sigma}\left[\bar{R}+6(\bar{\nabla} \sigma)^{2}\right] \cdot\left(\frac{1}{16 \pi G}-f \cdot \sigma\right) \\
& -\int \mathrm{d}^{4} x \sqrt{-\bar{g}} e^{4 \sigma} \cdot\left(\frac{\Lambda}{8 \pi G}-g \cdot \sigma\right),
\end{aligned}
$$

where the "massless" terms in the r.h.s. were defined in (4) and (11) and the coefficients $f$ and $g$ can be expressed via the dimensional parameters

$$
\begin{aligned}
\tilde{f} & =\frac{16 \pi f}{M_{P}^{2}} \\
& =\frac{1}{2 \pi} \sum_{\text {scalars }} \frac{N_{s} m_{s}^{2}}{M_{\mathrm{P}}^{2}}\left(\xi-\frac{1}{6}\right)+\frac{1}{3 \pi} \sum_{\text {fermions }} \frac{N_{f} m_{f}^{2}}{M_{\mathrm{P}}^{2}}
\end{aligned}
$$




$$
\begin{aligned}
\tilde{g} & =\frac{8 \pi g}{M_{P}^{2} \Lambda} \\
& =\frac{1}{4 \pi} \sum_{\text {scalars }} \frac{N_{s} m_{s}^{4}}{M_{\mathrm{P}}^{2} \Lambda}-\frac{1}{\pi} \sum_{\text {fermions }} \frac{N_{f} m_{f}^{4}}{M_{\mathrm{P}}^{2} \Lambda} .
\end{aligned}
$$

In the last expression we assume for simplicity that masses of all fermions are equal, and the same with scalars masses and non-minimal parameter $\xi$. The possibility of $\xi \neq 1 / 6$ is introduced for generality, more detailed discussion will be given in Sect. 5.

Equation (25) is not an exact result like (11), even for the FRW metric. The reason is that the conformal-invariant functional $S_{c}$ in this case depends not only on the metric $g_{\mu \nu}$, but also on the scalar field $\chi$. The approximation which provides Eq. (25) becomes clear if we remember that the renormalization group in curved space is related to the global rescaling of the metric, $g_{\mu \nu} \rightarrow g_{\mu \nu} \cdot \exp (2 \tau)$ [4,59-61]. Since coefficients $\omega, b, c, f, g$ are the Minimal Subtraction schemebased $\beta$-functions of the higher derivative parameters, $G^{-1}$ and $\rho_{\Lambda}=\Lambda / 8 \pi G$, it is easy to note that (25) is exactly the renormalization group improved classical action of vacuum (4), where the global scaling parameter is replaced by the time-dependent conformal factor of the metric, $\tau \rightarrow \sigma(t)$. Hence the approximation assumed in (25) is the one of the Minimal Subtraction scheme of renormalization. Within this approximation one cannot observe effects of masses, such as low-energy decoupling. However, it is a reliable approximation at high energies, including at the initial stage of the stable anomaly-driven inflation. ${ }^{3}$

Different from the effective action of massless fields [28, 31], the covariant version of Eq. (25) is not known, but this expression is sufficient for basic cosmological application. One can safely assume that the cosmological constant and its running do not play essential role at the inflation epoch. Following [42-44], we set $\Lambda=\tilde{g}=0$. Then the equation for $\sigma(t)$ is

$$
\begin{gathered}
\dddot{\sigma}+7 \dddot{\sigma} \dot{\sigma}+4 \ddot{\sigma}^{2}+4\left(3-\frac{b}{c}\right) \ddot{\sigma} \dot{\sigma}^{2}-\frac{4 b}{c} \dot{\sigma}^{4} \\
-\frac{M_{p}^{2}}{8 \pi c}\left[\left(\ddot{\sigma}+2 \dot{\sigma}^{2}\right)(1-\tilde{f} \sigma)-\frac{\tilde{f}}{2} \dot{\sigma}^{2}\right]=0 .
\end{gathered}
$$

The new part compared to Eq. (16) is the presence of the mass-dependent terms with $\tilde{f}$, also we use other variable, according to Eq. (12).

An approximate solution of Eq. (28) can be obtained by the replacement

$$
M_{\mathrm{P}}^{2} \longrightarrow M_{\mathrm{P}}^{2}[1-\tilde{f} \sigma(t)]
$$

\footnotetext{
3 This result of Refs. [42-44] concerning the effects of massive fields has been independently confirmed in [62] by technically different method (see also [57]).
}

in the expression for the Hubble parameter (18) corresponding to the massless solutions (17). The solution has the form

$\sigma(t)=H_{0} t-\frac{H_{0}^{2}}{4} \tilde{f} t^{2}$

which reproduces numerical solutions of (28) with a very good precision. Equation (30) describes a tempered form of inflation, which starts as an exponential (massless-based) version (17) and ends when $H$ decreases to the value where the decoupling of $s$-particles starts.

Starting from the solution (30), we need to know when the stable phase of inflation ends and what happens with the universe after that. If $H^{*}$ is the energy scale where the supersymmetry breaks down, the stability holds until the moment of time $t_{*}$, when $H\left(t_{*}\right)=H_{0}-(1 / 2) H_{0}^{2} \tilde{f} t_{*}=H^{*}$. This expression means that at the scale $H^{*}$ most of $s$-particles are beyond the IR cut-off, $M_{s}>H^{*}$ and decouple from gravity at the quantum level. After certain amount of such particles decouple, the sign of the $\beta$-function $c=\beta_{3}$ in (9) changes to the opposite and inflation gets unstable. It is a natural to suppose that $H^{*}$ should have the same magnitude as the mass scale of supersymmetry breaking, $M_{S U S Y}$. Another quantity which depends on the same scale is $\tilde{f} \sim\left(H^{*} / M_{P}\right)^{2}$.

The next issue to address is what happens to the universe after it goes through the critical point $H(t)=H^{*}$. Indeed, this question is very difficult to answer and hence we have to change the style of the consideration. Until this moment our consideration was based on the use of the QFT results, such as Eq. (28), even if the derivation of the equation required some risky methods such as conformal replacement of dimensional parameters [42]. Unfortunately, in the vicinity of the critical point $H=H^{*}$ the QFT provides no real help and even no insight. The reason is that we do not have an approximation or approach to deal with the situation when the Hubble parameter is of the same order of magnitude as the mass of the free quantum field on curved background. It is obvious that the usual expansion in powers of curvature tensor over square of mass of the quantum field has no much sense. On the other hand, the approach which led us to (28) is based on treating mass as a small perturbation and, therefore, also does not work for $H(t) \sim H^{*}$.

In the next two sections we will deal with this difficult problem in a most simple way, which can be called the "sharp cut-off" method. Namely, we consider the final point of the evolution (30) as a starting point of the unstable inflation. Of course, this is a great simplification, since we completely ignore the quantum effects in the intermediate epoch. In the "sharp cut-off" approach we simply cut-off the intermediatescale quantum effects and try to see in which part of the phase diagram of the unstable inflation we arrive by moving along the solution (30). 


\section{Numerical study of transition to unstable epoch}

Consider that the stable phase ends at the moment $t_{*}$ when the Hubble parameter has the value approximately corresponding to (27),

$H^{*}=H\left(t_{*}\right)=\sqrt{\tilde{f}} M_{\mathrm{P}}$.

Using the last equation and Eq. (30), the value of $t_{*}$ can be expressed as

$t_{*}=\frac{2}{H_{0}^{2} \tilde{f}}\left(H_{0}-\sqrt{\tilde{f}} M_{\mathrm{P}}\right)$.

The initial conditions for the consequent unstable evolution can be calculated through Eqs. (32) and (30), using the Minimal Supersymmetric Standard Model (MSSM) particle content, $N_{s, f, v}=(104,32,12)$. We assume that $\tilde{f}=10^{-4}$, which is close to the value for the GUT-scale supersymmetry.

An explanation of the choice of the value of $\tilde{f}$ is in order here. The GUT-scale supersymmetry is different from the usual $\mathrm{TeV}$-scale supersymmetry which is useful to address the gauge hierarchy problem. The unique aspect of supersymmetry which is relevant for us here is the change of sign in the relation (19) when the supersymmetry is broken, hence we are not confined by some specific scale of supersymmetry breaking. Looking at the definition (26) it is clear that the value corresponding to the GUT-scale supersymmetry should be $\tilde{f} \propto 10^{-6}$ and for the $T e V$-scale supersymmetry about $\tilde{f} \propto 10^{-32}$. Let us note that an inflationary model that pretends to describe the observed power spectrum of density perturbations (without inflaton) should have $H \approx 10^{14} \mathrm{GeV}$. Then $\tilde{f}$ should be no less than $10^{-10}$. Since for the GUT-scale SUSY one has $\tilde{f} \approx 10^{-6}$ and this is the mostly interesting case. We have checked numerically that the qualitative aspects of transition which will be discussed below are not sensible to the choice of $\tilde{f}$. For making plot and presentation in general better, we mainly use larger value of $\tilde{f}=10^{-4}$. This value also exceeds the value $\tilde{f} \approx 10^{-5}$ at which the comoving scale corresponding to the present Hubble radius first crossed it in the opposite direction during unstable inflation in the $R+R^{2}$ model; see Sect. 5 .

Let us present the results of the numerical solution of Eq. (16) in the unstable case with the initial data corresponding to the point $H^{*}$. For the sake of definitiveness, we consider the Minimum Standard Model (MSM) particle content $N_{s, f, v}=$ $(4,24,12)$.

The numerical solution at small scales of time show oscillations in the Hubble parameter $H$ as shown in Fig. 1.

Later on the amplitude of these oscillations becomes smaller and the Hubble parameter behavior starts to look very similar to the radiation-dominated universe without quantum

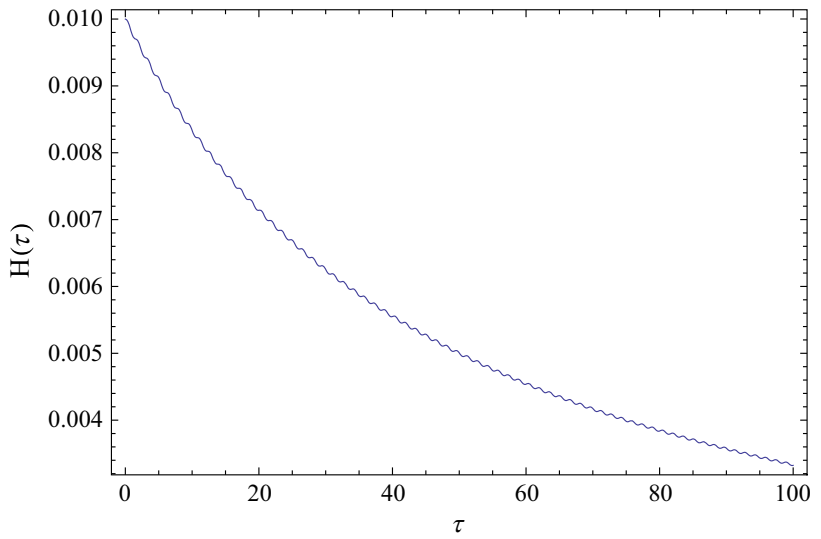

Fig. 1 Numerical solution for the Hubble parameter $H(t)$ in the units of Planck time $\tau \equiv t / t_{P}$ for the MSM particle content and $\tilde{f}=10^{-4}$

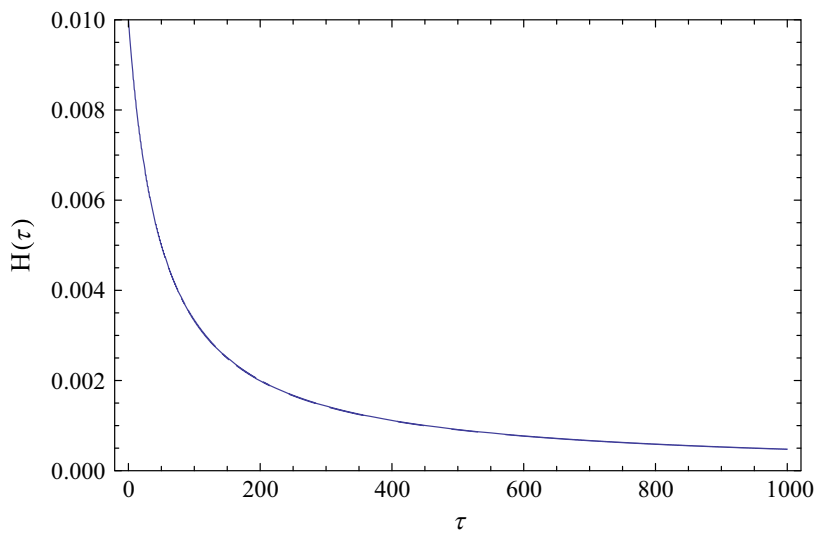

Fig. 2 The same case as in Fig. 1, but at larger scale. MSM and $\tilde{f}=$ $10^{-4}$

corrections $H(t) \sim 1 / 2 t$. The situation is illustrated in Figs. 1 and 2.

The oscillations which we observe in these plots can lead to the production of matter particles. But since the physical unstable inflation is still to come, this is not a physically relevant process. After a certain period corresponding to the MSM, the expansion of the universe becomes weaker and at some point even the contributions of massive nonsupersymmetric particles get decoupled. At the last stage only the massless particle - the photon, gives contributions. The numerical analysis for the unstable inflation driven by a single photon as an active quantum field, $N_{s, f, v}=(0,0,1)$ leads to the plots shown in Fig. 3. In this case one can observe, once again, some oscillations for the initial short period of time, however, the amplitude of the oscillations is weaker compared to the MSM case. And oscillations become weaker after the initial period.

The case of a single photon is an extra example of stabilizing perturbations. For the reasons explained above, it is not useful for describing inflation, and it is included mainly for generality. At the same time, it has some physical rele- 


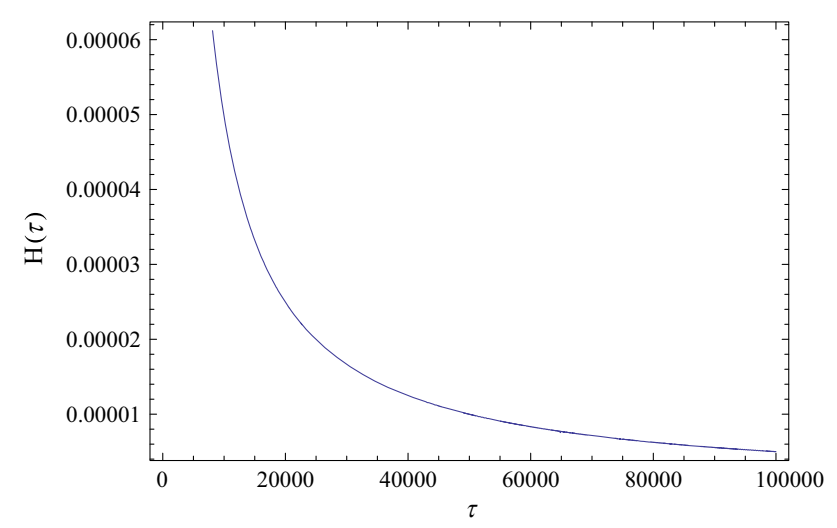

Fig. 3 Numerical solution for the Hubble parameter $H$ in units of Planck time $\tau \equiv t / t_{P}$ for the photon case and $\tilde{f}=10^{-4}$

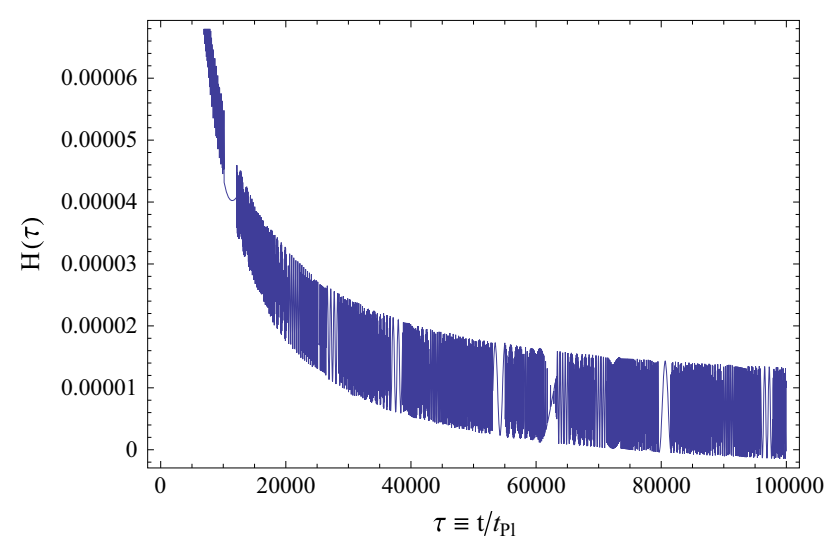

Fig. 4 Part of the plot from Figs. 1 and 2, but with much smaller scale in the $H$ axis

vance, not linked to inflation, but serving as a test for the consistency of the anomaly-induced model. Since we are dealing with the higher derivative action, it is important to ensure that the physically relevant solutions do not suffer from the Ostrogradsky-like instabilities. Looking backward to the history of the universe, the last strong perturbation for the conformal factor occurred at the epoch when quantum contributions of neutrino decouple from gravity. According to the plot of Fig. 3 the perturbations for the conformal factor are stabilized after this decoupling.

The difference between the photon case and the one of MSM is quantitative, namely oscillations have smaller amplitudes for a photon. In order to show that the oscillations still take place, we show a part of the previous plots with another scale in Fig. 4.

\section{Phase diagrams and stitching the solutions}

In this section we shall consider the transition between stable and unstable regimes by means of phase diagrams. Instead

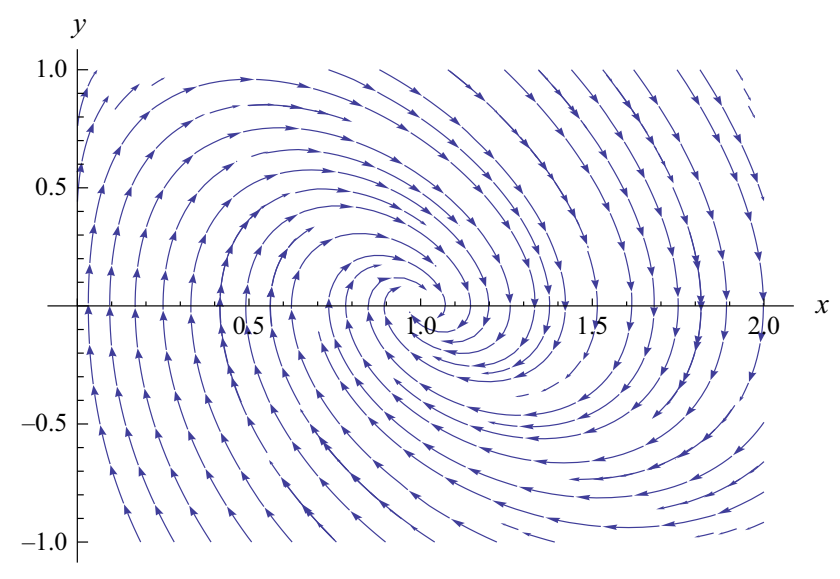

Fig. 5 Phase diagram of Eq. (35) with MSSM particle content

of Eq. (30) one can use 00-component of the equation

$R_{\mu \nu}-\frac{1}{2} g_{\mu \nu}(R-2 \Lambda)=8 \pi G\left\langle T_{\mu \nu}\right\rangle$,

in our case it has the form of the third-order equation

$\frac{2 \dot{a} \ddot{a}}{a^{2}}-\frac{\ddot{a}^{2}}{a^{2}}+\frac{2 \ddot{a} \dot{a}^{2}}{a^{3}}-\left(3+\frac{2 b}{c}\right) \frac{\dot{a}^{4}}{a^{4}}-\frac{M_{\mathrm{P}}^{2}}{8 \pi c} \frac{\dot{a}^{2}}{a^{2}}=0$.

Equation (34) can be reduced to the first-order differential equation

$\frac{\mathrm{d} y}{\mathrm{~d} x}=\frac{b\left(x-x^{-1 / 3}\right)}{6 c y}-1$

by the following change of variables $[15,16]$ :

$$
\begin{array}{r}
x=\left(\frac{H}{H_{0}}\right)^{3 / 2}, \\
y=\frac{\dot{H}}{H_{0}^{3 / 2}} H^{-1 / 2}, \\
\mathrm{~d} t=\frac{\mathrm{d} x}{3 H_{0} x^{2 / 3} y},
\end{array}
$$

where (as before) $H_{0}=M_{\mathrm{P}} / \sqrt{-16 \pi b}$.

The critical point $(1,0)$ corresponds to the exponential solution (17) in both stable and unstable cases. For a stable inflation $[43,44]$ based on the MSSM particle content, the phase diagram of Eq. 35 is shown in Fig. 5. As we can see, there is a single attractor corresponding to the inflationary solution (17).

For the unstable case we arrive at the phase diagram shown in Fig. 6. In this case there are different attractors $[15,16]$.

Let us see which integral curve in the unstable case of Fig. 6 corresponds to the initial point $x_{0}, y_{0}$ where the stable regime ends. Placing the solution (30) into expressions (36) 


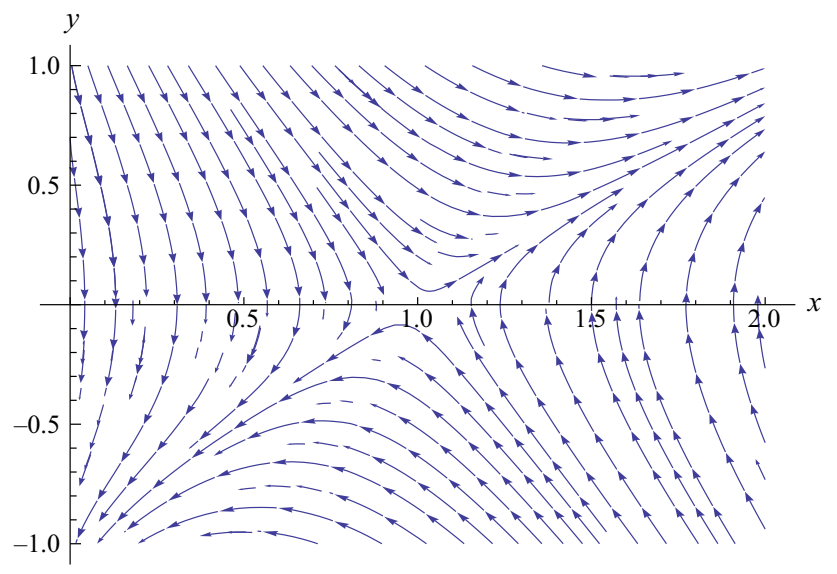

Fig. 6 Phase diagram of Eq. (35) with MSM particle content

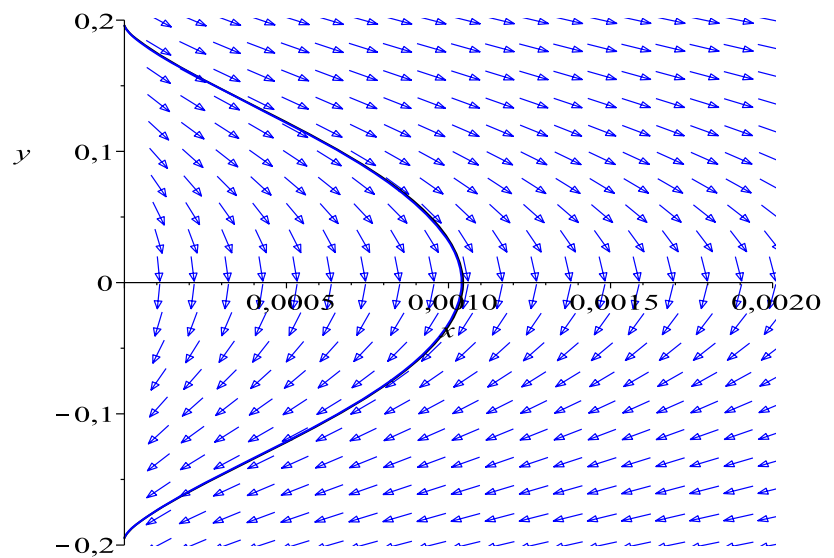

Fig. 7 Integral curve corresponding to Eq. (37) and $\tilde{f}=10^{-4}$

we find

$x_{0}=(-16 \pi b \tilde{f})^{3 / 4}, \quad y_{0}=-\frac{1}{4}\left(\frac{\tilde{f}^{3}}{-16 \pi b}\right)^{1 / 4}$.

Taking this initial value, one meets the integral curve shown in Fig. 7.

Let us say that this result nicely fits our most optimistic expectations. It exactly corresponds to the relatively "peaceful" unstable inflation qualitatively similar to the one of $[15,16]$, and not to the "hyperinflation"-type explosion, which was described in [35]. Anyway, the initial phase of such an inflation can be very violent, because the coefficient of the $R^{2}$ is very small. In the next section we describe how it can be enormously increased during the transition period, which was simply ignored until now within the "sharp cutoff" simplification.

\section{UV/IR running and generating huge $R^{2}$-term}

As we have seen in the previous sections, the end of the stable phase of inflation occurs in the region of the phase plane from which the universe can continue into the unstable phase. However, in order to have a successful inflationary model we need to go beyond the anomaly-induced effective action. The reason is that the coefficient of the overall $R^{2}$-term with a much greater value, about $5 \times 10^{8}$, is required to control density perturbations after the inflation period ends [26]. In more detail: the dimensionless coefficient in the action in front of the $R^{2}$ should be $N^{2} /\left(288 \pi^{2} A_{s}\right)$, where $A_{s}(k)$ is the amplitude of the power spectrum of primordial scalar (density) perturbations, while $N$ is the number of $e$-folds from the end of inflation and $\log \left(k_{\mathrm{fin}} / k\right)$ at the same time. ${ }^{4}$ $A_{s}(k)$ is also proportional to $N^{2}$ for the model involved. According to the most recent measurements [45] $A_{s}(k) \approx$ $2.2 \times 10^{-9}$ for $k=k_{0}=0.05 \mathrm{Mpc}^{-1}$. Choosing $N=55$ for $k=k_{0}$ we arrive at the estimate $5 \times 10^{8}$ for the coefficient of the $R^{2}$-term. Thus, it is the observed smallness of largescale inhomogeneous perturbations in the present universe (characterized by the small value of $A_{s}$ ) that requires the coefficient in front of the $R^{2}$ term to be large and of the order of $A_{s}^{-1}$ during the last, unstable and observable part of inflation. An alternative to this could be to add some other non-gravitational scalar field by hand which would support the second (unstable) part of inflation. The simplest models of such kind of double inflation were investigated in $[63,64]$. However, both models considered in these references use trans-Planckian values of this inflaton field and produce too large amount of primordial gravitational waves, which has been excluded by recent observational data [45].

Note that the same observational data on the power spectrum of primordial density perturbations in the universe show also that any higher-order terms of the type $R^{n}, n>2$ added to the action of the unstable anomaly-induced inflation are strongly (exponentially in $n$ ) suppressed for the number of e-folds from the end of inflation $N \lesssim 60$ [65]. Thus, observations demand the absence of significant higher-order in $R$ corrections to the phenomenological $R+R^{2}$ inflationary model that provides an independent support to theoretical arguments for the conservation of the structure of the conformal anomaly in higher loops discussed in Sect. 2.1.

Quantum decoupling of $s$-particles can explain the change of the sign of $c$, but cannot make it grow so much. The purpose of the present section is to discuss alternative mechanisms which can produce a dramatic change of the coefficient $c$ in the epoch close to the change of its sign. ${ }^{5}$ Let us stress that we have no reliable information as regards the physical theories at the GUT scale or even the supersymmetry breaking scale when the transition from stable to unstable versions of anomaly-driven inflation is supposed to occur. Hence we are not in a position to indicate a definite mechanism which

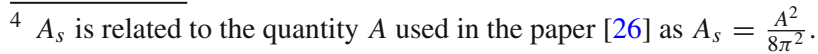

5 Recently another mechanism of generating a sufficiently large value of $a_{4}$ was discussed in [66] in the models with extra dimensions.
} 
provides such a dramatic growth of the coefficient of the $R^{2}$ term. Instead we shall describe two possible situations when such a growth is possible. In both cases the consideration is based on the relation between non-minimal scalar-curvature interaction and the vacuum $R^{2}$-term. This relation was previously discussed, e.g. in the context of supersymmetry [67].

\subsection{RG running of the non-minimal parameter $\xi$}

Renormalization group (RG) in curved space (see, e.g., [4]) tells us that the values of all parameters of the theory may run with change of energy scale. In particular, the RG for the coefficient $a_{4}$ in the vacuum action (4), (3) has a general form

$\mu \frac{\mathrm{d} a_{4}}{\mathrm{~d} \mu}=\beta_{4}=l_{1}+l_{2} \xi+l_{3} \xi^{2}$,

where the coefficients $l_{1,2,3}$ are given by power series in coupling constants, corresponding to the loop expansion. We assume that the high energy GUT-like model (supersymmetric or not) includes gauge $g$, Yukawa $h$, and four-scalar $f$ couplings, hence $l_{1,2,3}=l_{1,2,3}(g, h, f)$.

Indeed, all quantities in Eq. (38) are also running parameters and satisfy their own RG equation. In particular, the equation for $\xi$ has a general form

$\mu \frac{\mathrm{d} \xi}{\mathrm{d} \mu}=\beta_{\xi}=l_{4}+l_{5} \xi$,

where $l_{4,5}=l_{4,5}(g, h, f)$. In principle, the running (39) may significantly change both sign and magnitude of $\xi$, even at the short interval on the energy scale. The necessary condition for this intensive running is large values of at least some of the couplings $g, h, f$. This situation is possible near the transition, since it can be related to formation of condensate and then the non-perturbative regime may take place.

Note that at one loop the expressions are much simpler,

$\beta_{4}^{(1)}=l_{3}\left(\xi-\frac{1}{6}\right)^{2}$,

$\beta_{\xi}^{(1)}=\left(\xi-\frac{1}{6}\right) l_{5}$,

where

$l_{5} \sim l_{51} f+l_{52} h^{2}+l_{53} g^{2}$.

In the last formulas the coefficients $l_{3}, l_{51}, l_{52}, l_{53}$ are modeldependent constants.

At the one-loop order the conformal values $\xi=1 / 6$ and $a_{4}=0$ are fixed points, which can be stable in either UV or IR [68] (many examples and further references can be found in this work and in Ref. [4]). However, at higher loops the conformal value $\xi=1 / 6$ is not a fixed point, as was found for a scalar field in [69] and can also be established from a general considerations $[70,71]$.
It is natural, albeit not necessary, to assume that the value of $\xi$ in the far UV is conformal. 6 Suppose the "far UV" corresponds to the sub-Planckian energies. Then the value of $\xi$ can become very different from conformal already at the GUT scale, due to the running (39) in the framework of GUT theory. Hence when it comes to the transition from GUT to some lower energy theory, $\xi$ may be essentially away from the conformal point, even if it was at this point in the UV. Another important point is that around the scale of stable-unstable transition some of the interactions may become strong. Then, according to (39), the $\beta$-function for $\xi$ may be given by an infinite power series of large couplings. Assuming that this series is convergent, one can see that there is nothing wrong in a very intensive running of $\xi$ on a very short interval of the energy scale, before the masses of the fields grow large and running of $\xi$ stops due to the IR decoupling.

The next observation is that if $|\xi|$ becomes very big, then the coefficient $a_{4}$ can become even much greater, due to the quadratic dependence in (38), and especially assuming large values of couplings before the "confinement" of the GUT degrees of freedom and the non-perturbative nature of Eq. (38) in this situation. It is worth noticing that both $\xi$ and $a_{4}$ are not couplings in the semiclassical theory, hence there is no contradiction to have their values large, even within the perturbative approach. Indeed, these arguments can easily explain the value of $\xi \approx 40,000$, which is required for the Higgs inflation [72]. As well, or even more natural, these arguments can explain that the value of $a_{4}$ is about $5 \times 10^{8}$, which is roughly the square of the mentioned value of $\xi$.

It may be a very interesting problem to construct a model of GUT and its breaking into Standard Model plus a hidden Dark sector, which yields the picture described above. But since this consideration is beyond the scope of the present work, let us describe the second possibility to gain a very large value of the coefficient $a_{4}$ of the $R^{2}$-term.

\subsection{Spontaneous symmetry breaking (SSB) with non-zero $\xi$}

As a second example, we review how the $R^{2}$-term can emerge due to the spontaneous symmetry breaking (SSB) in the presence of non-minimal coupling between scalar field and scalar curvature, $\xi R \varphi^{2}$. Let us stress that a non-zero $\xi$ is a necessary condition of renormalizability of the theory with the Higgs field or its analogs in GUT models. Another question of whether the large value of $\xi$ is "natural".

As we discussed above, quantum corrections can produce an intensive running of $\xi$. Then, since $\xi$ is a dimensionless quantity, it cannot be regarded large or small by itself. In order to evaluate whether $\xi$ is large or not, one

\footnotetext{
${ }^{6}$ For instance, this is required by the field-particle correspondence (traceless $T_{\nu}^{\mu}$ ) for the effectively massless free fields, since we assume asymptotic freedom in the fundamental theory.
} 
has to compare the corresponding dimensional combinations with some reference quantity. In our case the comparison should be done between $\xi R$ and the square of mass $m$ of the scalar field, since they always emerge in a linear combination $\mp m^{2}+\xi R$ (the choice of a sign depends on whether the SSB is assumed or not). For instance, in the present-day universe and Higgs field the numbers are $m^{2}=m_{H}^{2} \propto 10^{4} \mathrm{GeV}^{2}$ and $R \sim H_{0}^{2} \propto 10^{-84} \mathrm{GeV}^{2}$ ( $H_{0}$ is the value of Hubble parameter). Obviously, the values of $\xi=10^{4}-10^{5}$ do not look large in this case. Indeed, the situation may be different in the early universe, since the curvature has been much greater then. In this situation the curvature effects can be relevant for the SSB and Higgs, which is well known from the studies of curvature-induced phase transitions (see, e.g., [4]) and Higgs inflation [72-77].

Let us consider how the $R^{2}$-term emerges in the induced action of gravity in the theory with non-minimal interaction $\xi$ and SSB. The considerations presented below are not directly related to the Higgs field in the Standard Model and can also be applied to more general theories at different energy scales.

We start by briefly reviewing SSB in curved space-time, in the way it was originally discussed in [78]. Consider the classical action of a scalar field $\varphi$,

$$
\begin{aligned}
S= & \int \mathrm{d}^{4} x \sqrt{-g}\left\{g^{\mu \nu} \partial_{\mu} \varphi^{*} \partial_{\nu} \varphi+\mu_{0}^{2} \varphi^{*} \varphi\right. \\
& \left.+\xi R \varphi^{*} \varphi-\lambda\left(\varphi^{*} \varphi\right)^{2}\right\} .
\end{aligned}
$$

The vacuum expectation value $v$ for the scalar field is defined by the relation

$$
-\square v+\mu_{0}^{2} v+\xi R v-2 \lambda v^{3}=0 .
$$

For a minimal interaction case $\xi=0$ we have a constant solution,

$v_{0}^{2}=\frac{\mu_{0}^{2}}{2 \lambda}$

However, in a generic curved space one cannot find a constant solution due to the potentially variable curvature scalar. Then the $\square$-term in (44) cannot be neglected and a closed-form compact solution is impossible. At the same time, one can obtain a solution in the form of the power series in $\xi$,

$v(x)=v_{0}+v_{1}(x)+v_{2}(x)+\cdots$,

regarding (45) as a zeroth-order approximation.

In the first order we find [78]

$v_{1}=\frac{\xi v_{0}}{\square-\mu^{2}+6 \lambda v_{0}^{2}} R=\frac{\xi v_{0}}{\square+4 \lambda v_{0}^{2}} R$.
It is not difficult to derive the next orders of this expansion, for instance

$$
\begin{aligned}
v_{2}= & \frac{\xi^{2} v_{0}}{\square+4 \lambda v_{0}^{2}}\left\{R \frac{1}{\square+4 \lambda v_{0}^{2}} R\right. \\
& \left.-6 \lambda v_{0}^{2}\left(\frac{1}{\square+4 \lambda v_{0}^{2}} R\right)^{2}\right\} .
\end{aligned}
$$

In the points of the minima we meet the induced gravity action. Replacing the solution (46) into the scalar action (43), we obtain the induced low-energy action of vacuum, depending only on the metric,

$$
\begin{aligned}
S_{\text {ind }}= & \int \mathrm{d}^{4} x \sqrt{-g}\left\{g^{\mu v} \partial_{\mu} v \partial_{\nu} v\right. \\
& \left.+\left(\mu_{0}^{2}+\xi R\right) v^{2}-\lambda v^{4}\right\} .
\end{aligned}
$$

Making an expansion in the powers of $\xi$, at the second order we arrive at the expression

$$
\begin{aligned}
S_{\text {ind }}= & \int \mathrm{d}^{4} x \sqrt{-g}\left\{-v_{1} \square v_{1}+\xi R\left(v_{0}^{2}+2 v_{0} v_{1}\right)\right. \\
& +\mu^{2}\left(v_{0}^{2}+2 v_{0} v_{1}+2 v_{0} v_{2}+v_{1}^{2}\right) \\
& \left.-\lambda\left(v_{0}^{4}+4 v_{0}^{3} v_{1}+4 v_{0}^{3} v_{2}+6 v_{0}^{2} v_{1}^{2}\right)\right\}+\mathcal{O}\left(R^{3}\right) \\
= & \int \mathrm{d}^{4} x \sqrt{-g}\left\{\lambda v_{0}^{4}+\xi R v_{0}^{2}\right. \\
& \left.+\xi^{2} v_{0}^{2} R \frac{1}{\square+4 \lambda v_{0}^{2}} R+\cdots\right\} .
\end{aligned}
$$

The first two terms in the last action represent an induced cosmological constant (CC) and Einstein-Hilbert terms, respectively.

Let us make a few observations concerning the last result (49). The induced CC density in the first term is huge compared to the observed value, hence the compensating vacuum $\mathrm{CC}$ should be introduced. There is an extensive discussion of the fine-tuning required for this compensation; see, e.g., the standard review [79] and recent work treating this problem in the QFT framework [80,81]. The second term in (49) is the induced Einstein-Hilbert term. For $\xi v_{0}^{2} \ll M_{P}^{2}$ the value of the coefficient of this term is not sufficient to have a purely induced gravity, hence the corresponding vacuum term is required. In the case of the Standard Model Higgs and $\xi \propto 10^{4}$ the induced term is just a very small correction to the vacuum term. One can say that the situation is opposite to the one with the $\mathrm{CC}$ term.

The third term in (49) is quadratic in scalar curvature and in $\xi$ and it is non-local. It is easy to see that this term behaves in a very different way in the UV and IR limits. The definition of UV here is related to the magnitude of derivatives of the curvature tensor, compared to $v_{0}^{2}$. In the case when $\square R \gg v_{0}^{2} R$, the term is essentially non-local and shows the global scaling which is identical to the one of the EinsteinHilbert term. Let us mention, in passing, that the next local 
term, of the $R\left(\square+4 \lambda v_{0}^{2}\right)^{-2} R$-type, cancels identically, hence there is no similar correction to the CC-term from $\mathrm{SSB}^{7}$. For inflation, when $R$ is approximately constant, we can assume an opposite relation $\square R \ll v_{0}^{2} R$. Then the third term in (49) becomes effectively local and equal to

$S_{\text {ind }}=\frac{\xi^{2}}{4 \lambda} \int \mathrm{d}^{4} x \sqrt{-g} R^{2}$.

One can note that in the same approximation the next-order terms are suppressed by higher powers of $\xi R / v_{0}^{2}$ and therefore the last term represents the leading quantum contribution with higher powers of scalar curvature being small corrections to it.

One can easily see that the term (50) is close to what we need for a successful "jump" in the value of the coefficient of the $R^{2}$-term due to the phase transition related to SSB. Assuming that $\xi$ has a large value, e.g., due to the mechanism which we discussed in the previous subsection, the small value of the four-scalar coupling $\lambda$ in the IR is enhancing the effect of running.

Different from the running of $a_{4}$, the induced value (50) has a definite positive sign. According to Eqs. (9), (19), and (11) this is the same sign which we need for the unstable $R+R^{2}$ model $[15,16]$. In the GUT-like models with several scalars there are typically different $\lambda$ and $\xi$ for each of these scalars. Then it is sufficient that one of the combinations $\xi^{2} / \lambda$ becomes very large at the instant when the scalar is freezing in the vacuum state, to provide a desired huge value of induced coefficient $a_{4}$. It would be definitely interesting to construct an explicit realization of this situation in the framework of some GUT-like model.

Unfortunately the consideration presented above does not work for the $R^{2}$-inflation [15], because during inflation the magnitude of the product $\xi R$ is too large and the expansion (46) is not appropriate. ${ }^{8}$ One of the possibilities is to consider a different expansion using a de Sitter starting point $\mu_{0}^{\prime 2}=$ $\mu_{0}^{2}+\xi R$ instead of $\mu_{0}^{2}$. However, this approach also meets a serious problem.

It is worthwhile to discuss the situation in detail and see if the appearance of the large $R^{2}$ term can be explained by the modified SSB for a sufficiently low value of the Ricci scalar $R$. This value should be still sufficient for the unstable $R^{2}$ inflation to occur with the parameter $a_{4}$ following from observational data. For a slow-roll quasi-de Sitter inflation, when $R<0$ can be considered as a constant in the zero

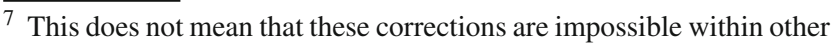
approaches. There was recently an interesting work [82] (see further references therein) about the cosmological relevance of the massless version of such a term, proposed originally in [78].

8 We are grateful to the anonymous referee for indicating this important point to us.
}

approximation, the non-trivial solution of Eq. (44) is

$v_{0}^{\prime 2}=\frac{\mu_{0}^{2}+\xi R}{\lambda}$.

Thus, if we want to have symmetry restored at large $|R|$ and $R<0$, we have to take $\xi>0$, i.e., the same sign of $\xi$ as in the case of conformal coupling $\xi_{\text {conf }}=1 / 6$ and opposite to that for the Higgs inflationary model [72]. Then the SSB occurs for $|R|<\mu_{0}^{2} / \xi$, and the value of $v_{0}^{\prime}$ is given by Eq. (51).

Replacing Eq. (51) into the action (43) and adding the Einstein term, we get the effective Lagrangian density in the quasi-static (slow-roll) case:

$L=-\frac{M_{\mathrm{P}}^{\prime 2} R}{2}+\frac{\xi \mu_{0}^{2} R}{2 \lambda}+\frac{\xi^{2} R^{2}}{4 \lambda}$

where $M_{\mathrm{P}}^{\prime}=(8 \pi G)^{-1 / 2}$ is the reduced Planck mass. In this model, the unstable $R^{2}$ inflation occurs for values of scalar curvature $|R| \gg R_{I}=\lambda M_{[\mathrm{P}]}^{\prime} / \xi^{2}$. Thus, $\lambda / \xi^{2}$ should be small to justify quasi-classical description of space-time. This condition can easily be achieved. However, the condition that the SSB occurs at curvatures $|R| \gg R_{I}$ requires also

$\mu_{0}^{2} \gg \xi R_{I}=\frac{\lambda M_{\mathrm{P}}^{\prime 2}}{\xi}$.

Then the coefficient in front of $R$ in Eq. (52) has the wrong, positive sign (i.e. gravity becomes repulsive at low curvature, in particular, in almost flat space-time). Therefore, we come to conclusion that it is not possible to use this type of SSB to generate the large coefficient $a_{4}$ needed for viable unstable inflation. At the same time, there is a chance that some other modified scheme related to a phase transition may be working.

\subsection{Unstable phase with a large $R^{2}$-term}

Assuming that there is a desired increase of the value of $a_{4}$ in the transition period, it is natural to ask how it will change the evolution if the universe in the consequent unstable period of inflation. In order to see this, we repeated the analysis of Sects. 3 and 4 , but this time with a very large value of $a_{4}=5 \times 10^{8}$.

Qualitatively, the phase diagram does not change too much, since the system is still in the "right" part of the phase plane of Fig. 6. The plot for the trajectory is similar to the one in Fig. 7, but now much closer to the origin of coordinates $x$ and $y$.

The result of numerical analysis is shown in Fig. 8. It is easy to see that the dynamical system of our interest suffers from initial oscillations which last a very short period. After that the dependence looks linear. 


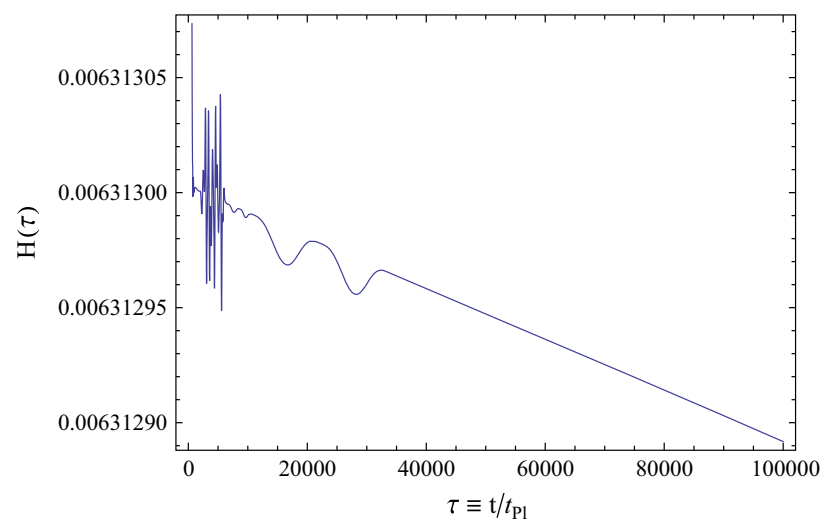

Fig. 8 The plot of $H(t)$ for the theory with one photon and $a_{4}=5 \times 10^{8}$

The interpretation of the plot in Fig. 8 is straightforward. The initial very fast change of $H(\tau)$ represents a remnant of the stable phase, with a relatively large initial value of $H$. After that the huge $R^{2}$-term starts to dominate. After some oscillations the universe starts the period of unstable inflation in the $R+R^{2}$-model. The analytic expression for this phase is very similar to Eq. (30),

$\sigma(t)=H_{1} t-\frac{M^{2}}{12} t^{2}+\mathcal{O}\left(\ln \left(t_{f}-t\right)\right)$,

where $t_{f}$ corresponds to the end of inflation, $H_{1}$ is an integration constant and $M \ll H$ in the given phase [26]. Equations (30) and (54) both lead to the approximately linear time dependence of $H$, which we can also observe in the plot under discussion.

It looks like the transition from stable to unstable phases is rather successful in the presence of a huge $R^{2}$-term, since it leads to the known dynamics after this transition, and this is exactly the dynamics which passed some tests in comparison with observational data. After all, the distinguished feature of the inflationary model based on the stable/unstable transition is the presence of preliminary stable phase. The consequences may be not observable, but this phase provides right initial conditions for the physically testable unstable phase.

\section{Conclusions}

The anomaly-driven inflation $[15,16]$ can be stable or unstable depending on the particle content of the underlying quantum field theory. In the course of inflationary expansion the number of the "active" fields can change, especially due to the quantum decoupling of heavy particles from gravity $[40,41]$. For example, one can expect the transition from stable to unstable phases in the supersymmetric versions of the Standard Model or GUTs, due to the decoupling of the $s$-particles.
The detailed description of the transition period is far beyond the available theoretical methods of quantum field theory, because this situation requires the description of the vacuum quantum effects in the case when Hubble parameter is of the same order of magnitude as the mass of the quantum massive field. For this reason, we use the most simple phenomenological approach to this problem, by assuming that the unstable part starts exactly in the point when the stable phase ends. The unstable particle contents may lead to the graceful exit to the usual radiation-dominated evolution, or to a very violent "hyperinflation"-like behavior $[15,17,35]$. By using numerical methods and also by stitching the solutions on the phase diagrams of the theory we have found that the point when the stable evolution ends exactly corresponds to the initial data of the desirable type of unstable evolution, such that the "hyperinflation"-type solutions are ruled out.

In order to have a phenomenologically successful $R+R^{2}$ inflation one need to explain also a relatively large value of the coefficient $a_{4}$ of the $R^{2}$-term. We discussed this issue starting from the renormalization group running of the nonminimal parameter $\xi$ and using two alternative ways to generate a huge $R^{2}$-term. The conclusion is that both mechanisms, namely the renormalization group running of vacuum $a_{4}$ and the SSB-based induced gravity, are capable to provide the coefficient of the $R^{2}$-term in the desirable range. The most important ingredient in both cases is a large value of the nonminimal parameter $\xi \propto 10^{4}$. From the QFT side, this means that it would be interesting to design the field theory models which could provide an intensive running of $\xi$ from the UV to IR, in either perturbative or non-perturbative frameworks. The first step in the non-perturbative direction has been done recently in [83], and the considerations presented above show that this is a phenomenologically relevant subject.

Qualitatively, the output of our work means that the transition from stable to unstable version of anomaly-driven inflation can occur successfully, at least it is so within the sharp cut-off approximation. In this case no special conditions for the initial data are required in the model. All these statements correspond to the dynamics of the conformal factor of the metric. It would be very interesting to extend it further to the case of initially anisotropic metrics. There is a strong expectation that anisotropy disappears rapidly during the stable phase, but this feature still requires a detailed investigation ([58]).

Acknowledgments T.P. is grateful to CAPES for supporting his Ph.D. project. I.Sh. was partially supported by CNPq, FAPEMIG and ICTP. The work of A.S. in Russia was supported by the RSF Grant 16-1210401. His visit to the Utrecht University was supported by the Delta ITP Grant BN.000396.1. He thanks Profs. S. Vandoren and T. Prokopec and Drs. J. van Zee for hospitality during the visit.

Open Access This article is distributed under the terms of the Creative Commons Attribution 4.0 International License (http://creativecomm ons.org/licenses/by/4.0/), which permits unrestricted use, distribution, 
and reproduction in any medium, provided you give appropriate credit to the original author(s) and the source, provide a link to the Creative Commons license, and indicate if changes were made.

Funded by SCOAP ${ }^{3}$.

\section{References}

1. A.A. Grib, S.G. Mamaev, V.M. Mostepanenko, Quantum Effects in Intensive External Fields (Atomizdat, Moscow, 1980). (In Russian)

2. A.A. Grib, S.G. Mamaev, V.M. Mostepanenko, Vacuum Quantum Effects in Str Fields (Friedmann Lab. Publ., St. Petersburg, 1994. (Modified translation to English)

3. N.D. Birell, P.C.W. Davies, Quantum Fields in Curved Space (Cambridge University Press, Cambridge, 1982)

4. I.L. Buchbinder, S.D. Odintsov, I.L. Shapiro, Effective Action in Quantum Gravity (IOP Publishing, Bristol, 1992)

5. L. Parker, D. Toms, Quantum Field Theory in Curved Spacetime: Quantized Fields and Gravity (Cambridge Univ. Press, Cambridge, 2009)

6. I.L. Shapiro, Class. Quant. Grav. 25, 103001 (2008). arXiv:0801.0216

7. I.L. Shapiro, PoS-JHEP 03, 1 (2006). arXiv:hep-th/0610168

8. M.J. Duff, Nucl. Phys. B 125, 334 (1977)

9. S. Deser, M.J. Duff, C. Isham, Nucl. Phys. B 111, 45 (1976)

10. Ya.B. Zeldovich, A.A. Starobinsky, Sov. Phys. JETP 34, 1159 (1972)

11. L. Parker, S.A. Fulling, Phys. Rev. D 9, 341 (1974)

12. M.J. Duff, Class. Quant. Grav. 11, 1387 (1994). arXiv:hep-th/9308075

13. P.C.W. Davies, Phys. Lett. D64, 402 (1977)

14. M.V. Fischetti, J.B. Hartle, B.L. Hu, Phys. Rev. D 20, 1757 (1979)

15. A.A. Starobinsky, Phys. Lett. B 91, 99 (1980)

16. A.A. Starobinsky, Nonsingular model of the universe with the quantum-gravitational de Sitter stage and its observational consequences. In: Proc. of the Second Seminar "Quantum Theory of Gravity" (Moscow, 13-15 October 1981) (INR Press, Moscow, 1982), pp. 58-72

17. A. Vilenkin, Phys. Rev. D 32, 2511 (1985)

18. P. Anderson, Phys. Rev. D 28, 271 (1983)

19. P. Anderson, Phys. Rev. D 29, 615 (1984)

20. P. Anderson, Phys. Rev. D 29, 1567 (1986)

21. M.B. Mijić, M.S. Moris, W.-M. Suen, Phys. Rev. D 34, 2934 (1986)

22. W.-M. Suen, P.R. Anderson, Phys. Rev. D 35, 2940 (1987)

23. S.W. Hawking, T. Hertog, H.S. Real, Phys. Rev. D 63, 083504 (2001)

24. V.F. Mukhanov, G.V. Chibisov, JETP Lett. 33, 532 (1981)

25. A.A. Starobinsky, JETP Lett. 34, 438 (1981)

26. A.A. Starobinsky, Sov. Astron. Lett. 9, 302 (1983)

27. A.A. Starobinsky, JETP Lett. 30, 682 (1979)

28. R.J. Riegert, Phys. Lett. B 134, 56 (1980)

29. E.S. Fradkin, A.A. Tseytlin, Phys. Lett. B 134, 187 (1980)

30. I. Antoniadis, E. Mottola, Phys. Rev. D 45, 2013 (1992)

31. I.L. Shapiro, A.G. Jacksenaev, Phys. Lett. B 324, 284 (1994)

32. P.O. Mazur, E. Mottola, Phys. Rev. D 64, 104022 (2001). arXiv:hep-th/0106151

33. A.M. Polyakov, Phys. Lett. B 103, 207 (1981)

34. I.L. Buchbinder, S.D. Odintsov, I.L. Shapiro, Phys. Lett. B 162, 92 (1985)

35. J.C. Fabris, A.M. Pelinson, I.L. Shapiro, Grav. Cosmol. 6, 59 (2000)

36. J.C. Fabris, A.M. Pelinson, I.L. Shapiro, Nucl. Phys. B 597, 539 (2001)

37. V. Müller, H.-J. Schmidt, A.A. Starobinsky, Phys. Lett. B 202, 198 (1988)
38. I.L. Shapiro, Int. J. Mod. Phys. D 11, 1159 (2002). arXiv:hep-ph/0103128

39. T. Appelquist, J. Carazzone, Phys. Rev. D 11, 2856 (1975)

40. E.V. Gorbar, I.L. Shapiro, J HEP 02, 021 (2003). arXiv:hep-ph/0210388

41. E.V. Gorbar, I.L. Shapiro, JHEP 06, 004 (2003). arXiv:hep-ph/0303124

42. I.L. Shapiro, J. Solà, Phys. Lett. B 530, 10 (2002)

43. A.M. Pelinson, I.L. Shapiro, F.I. Takakura, Nucl. Phys. B 648, 417 (2003). arXiv:hep-ph/0208184

44. A.M. Pelinson, I.L. Shapiro, F.I. Takakura, Nucl. Phys. B (PS) 127, 182 (2004). arXiv:hep-ph/0311308

45. P.A.R. Ade et al., Planck 2015 Results. XIII. Cosmological Parameters. arXiv: 1502.01589

46. J.C. Fabris, A.M. Pelinson, F.O. Salles, I.L. Shapiro, JCAP 02, 019 (2012). arXiv: 1112.5202

47. F.O. de Salles, I.L. Shapiro, Phys. Rev. D 89, 084054 (2014) arXiv: 1401.4583

48. M. Asorey, E.V. Gorbar, I.L. Shapiro, Class. Quant. Grav. 21, 163 (2003)

49. S.M. Christensen, Phys. Rev. D 17, 946 (1978)

50. J.S. Dowker, R. Chritchley, Phys. Rev. D 16, 3390 (1976)

51. Z. Komargodski, A. Schwimmer, JHEP 1112, 099 (2011). arXiv:1107.3987 [hep-th]

52. Z. Komargodski, JHEP 1207, 069 (2012). arXiv: 1112.4538 [hepth]

53. M.A. Luty, J. Polchinski, R. Rattazzi, JHEP 1301, 152 (2013). arXiv: 1204.5221 [hep-th]

54. A.M. Pelinson, I.L. Shapiro, Phys. Lett. B 694, 467 (2011). arXiv: 1005.1313

55. S.G. Mamaev, V.M. Mostepanenko, Sov. Phys. JETP 51, 9 (1980)

56. R.D. Peccei, J. Solà, C. Wetterich, Phys. Lett. B 195, 183 (1987)

57. A. Codello, G. D’Odorico, C. Pagani, R. Percacci, Class. Quant. Grav. 30, 115015 (2013). arXiv: 1210.3284

58. I.L. Shapiro, J. Solà, JHEP 02, 006 (2002)

59. B.L. Nelson, P. Panangaden, Phys. Rev. D 25, 1019 (1982)

60. I.L. Buchbinder, Theor. Math. Phys. 61, 393 (1984)

61. D.J. Toms, Phys. Lett. B 126, 37 (1983)

62. H.J. de Vega, N.G. Sanchez, Dark energy is the cosmological quantum vacuum energy of light particles. In: The Axion and the Lightest Neutrino. arXiv:astro-ph/0701212

63. L.A. Kofman, A.D. Linde, A.A. Starobinsky, Phys. Lett. B 157, $361(1985)$

64. S. Gottlöber, V. Müller, A.A. Starobinsky, Phys. Rev. D 43, 2510 (1991)

65. Q.-G. Huang, JCAP 1402, 035 (2014). arXiv:1309.3514

66. T. Asaka, S. Iso, H. Kawai, K. Kohri, T. Noumi, T. Terada, Reinterpretation of the Starobinsky Model. arXiv:1507.04344

67. S.V. Ketov, A.A. Starobinsky, JCAP 1208, 022 (2012). arXiv: 1203.0805

68. I.L. Buchbinder, I.L. Shapiro, E.G. Yagunov, Mod. Phys. Lett. A 5, 1599 (1990)

69. S.J. Hathrell, Ann. Phys. 139, 136 (1982)

70. G. de Berredo-Peixoto, E.V. Gorbar, I.L. Shapiro, Class. Quant. Grav. 21, 2281 (2004). arXiv:hep-th/0311229

71. M. Asorey, G. de Berredo-Peixoto, I.L. Shapiro, Phys. Rev. D 74, 124011 (2006). arXiv:hep-th/0609138

72. F.L. Bezrukov, M. Shaposhnikov, Phys. Lett. B 659, 703 (2008)

73. A.O. Barvinsky, A. Yu, A.A. Kamenshchik, Starobinsky. JCAP 0811, 021 (2008). arXiv:0809.2104

74. A.O. Barvinsky, A.Y. Kamenshchik, C. Kiefer, A.A. Starobinsky, C. Steinwachs, JCAP 0912, 003 (2009). arXiv:0904.1698

75. A.O. Barvinsky, A.Y. Kamenshchik, C. Kiefer, A.A. Starobinsky, C. Steinwachs, Eur. Phys. J. C 72, 2219 (2012). arXiv:0910.1041

76. F.L. Bezrukov, M. Shaposhnikov, JHEP 0907, 089 (2009). arXiv:0904.1537 
77. F.L. Bezrukov, A. Magnin, M. Shaposhnikov, Phys. Lett. B 675, 88 (2009). arXiv:0812.4950

78. E.V. Gorbar, I.L. Shapiro, JHEP 0402, 060 (2004). arXiv:hep-ph/0311190

79. S. Weinberg, Rev. Mod. Phys. 61, 1 (1989)

80. I.L. Shapiro, J. Solà, JHEP 0202, 006 (2002)
81. I.L. Shapiro, J. Solà, Phys. Lett. B 682, 105 (2009)

82. M. Maggiore, Dark Energy and Dimensional Transmutation in $R^{2}$ Gravity. arXiv:1506.06217

83. I.L. Shapiro, P.M. Teixeira, A. Wipf, Eur. Phys. J. C 75(6), 262 (2015). arXiv: 1503.00874 